\title{
Article title: The role of culture in relation to the seasonal influenza prevention practices of Hong Kong Chinese parents with preschool children
}

\section{Introduction}

In Hong Kong, seasonal influenza is common in the periods from January to March and July to August. Influenza is easily transmitted and particularly affects those with immature or compromised immune systems such as young children and the elderly with chronic illness (World Health Organization 2009; Centre of Health Protection of Hong Kong 2016). Hong Kong Chinese parents have been found to use both Western medicine and Traditional Chinese medicine remedies (Lau et al. 2005) to manage their children's flu symptoms and prevent their children from seasonal influenza infections. These include the use of herbal preparations such as Sang Ju Yin, Yu Ping Feng San, Folium Isatidis and Radix Scutellariae (Lau et al. 2005).

Differences in the health practices of families in Western and Asian societies have been noted in the literature (Joo 2014; Yamamoto and Li 2012). Individual and family health knowledge and practices, including health literacy are affected and shaped by culturally related principles and norms (Adair et al. 2004; Christensen 2004). For example, diet therapy, which is based upon a cultural belief in the energy giving effects of food is used in many Chinese families to achieve health to maintain balance by "heating" or "cooling" the body (Chen 2001). While foods such as honey or lemon drinks that seem to have soothing properties are often used in an attempt to prevent flu symptoms in Western families (Paul et al. 2007).

Hong Kong is highly westernized but the population is largely Chinese. Traditional Chinese Medicine and Western medicine continue to be integrated into long established health practices 
(Lam 2001). Traditional Chinese medicine includes different types of treatments such as herbal preparations, diet therapy, the use of animal organs, massage to stimulate blood circulation and acupuncture to treat a range of conditions (Ludman and Newman 1984). The principles underpinning Traditional Chinese Medicine and Western medicine are very different (Lam 2001). Chinese medicine is informed by the philosophical, ethical and religious tradition of Taoism that emphasizes harmony between human beings and nature providing peace of mind (Chen 2001). To attain health, an individual must regulate him or herself to fit the rhythms of the universe. The concepts of health and illness among Chinese people have long been influenced by the harmony of the forces of Yin and Yang (Chen 2001). The imbalance of these forces within and beyond the human body and its atmosphere is believed to cause illness. In contrast to Chinese medicine, Western medicine is based on the use of empirical or measurable evidence derived from the biomedical sciences, including genetics and involves medical technology to diagnose, treat, and prevent injury and disease.

The Hong Kong local health authorities have employed social marketing campaigns to promote personal and environmental hygiene strategies to prevent seasonal influenza. In addition, resources have been targeted at increasing immunization rates through vaccination programmes (Centre for Health Promotion of Hong Kong 2010). Maintaining personal hygiene including proper hand washing and cough etiquette, and having a seasonal influenza vaccination are recommended as the most effective methods to prevent the spread of seasonal influenza (World Health Organization 2009; Centre of Health Protection of Hong Kong 2014a; Jennings et al. 2008). 
Hong Kong is a small densely populated territory of over seven million people (Hong Kong Census and Statistics Department 2012). It is common for a family of four to live in an apartment with a total area of 40 square metres. Chinese values emphasize the development of interdependence and family closeness (Chen 2001), these beliefs also influence parents' childrearing practices such as sharing beds. In Hong Kong and many Asian countries, child-parent bed sharing is commonly observed in families (Yang and Hahn 2002; Liu, Liu, and Wang 2003). Under these circumstances, if a family member is infected with seasonal influenza, it is easily spread to other family members. The use of anticipatory interventions become crucial in reducing the risk of cross infection. These interventions include on-going strategies to maintain healthy immune responses and personal hygiene. Hong Kong Chinese mothers are highly involved in child care (Chan, Bowes, and Wyver 2009). As children often stay at home with their mothers during their early years, mothers are in a strong position to reinforce children's health practices through role modelling behaviours during daily interaction and providing timely reminders (Bodrovea and Leong 2007).

A review of the literature on seasonal influenza prevention, found that most research studies focus on the factors affecting vaccination uptake such as: influenza infection knowledge (Hofstetter, Barrett, and Stockwell 2015; Lohiniva et al. 2014; Liao et al. 2011); or perceived benefit from flu vaccination (Lau et al. 2012; Kwong, Lam, and Chan 2009; Zijtregtop et al. 2009); methods to increase influenza vaccination uptake rate (Stockwell et al. 2015); and the use of face masks and effective hand hygiene to prevent household influenza infection (Cowling et al. 2009). There are limited research studies exploring the role of culture in seasonal influenza prevention practices of Hong Kong Chinese parents with young children at a household level. The aim of this study is to provide a greater understanding of the role of culture in Chinese parents' health practices to prevent 
influenza. The findings will be of use for nurses and other community health professionals when working with Chinese communities in planning health promotion programmes and influenza preparedness plans.

\section{Methods}

\section{Research Design}

We undertook a mixed methods research study using a multiple-case approach (Cresswell 2009; Yin 2012). A case comprised a parent with a child between three-to-five years from a range of Hong Kong preschools and family backgrounds. This study approach enabled the researchers to: examine the phenomena from multiple views within and across each real-life setting, analyse the commonalities and variances between the cases and, discover previously unknown features regarding the culturally specific health promotion practices of parents related to seasonal influenza prevention (Yin 2014; Stake 1995).

\section{Recruitment and Participants}

A networking approach was used to identify potential preschools. Letters and study information sheets were delivered to parents via the principals of five kindergartens that invited the parents to join the study by contacting the researcher. Three kindergartens agreed to facilitate contact with potential parents. Parents indicating a willingness to communicate and share their experiences were then contacted by the researcher and a detailed explanation of the research was provided. Purposive intensity sampling was used to recruit parents. In total, 23 parents were contacted and 20 parents with healthy young children from 3-to-5 years agreed to participate in this study. 


\section{Data Collection}

Semi-structured interviews using open and closed questions were conducted in parents' homes. Digitally recorded interviews were transcribed verbatim for data analysis, with the aim of understanding parental health promotion strategies for seasonal influenza prevention and their health promotion practices to minimize the impact of flu symptoms or their onset. Open questions included: "can you describe how your family/cultural practices affect your decision-making about the management of seasonal influenza"; and "do any of your family members use alternative medicine to protect your child from seasonal influenza"? Demographic data were collected including household surface area, number of people living there and the child's sleeping arrangements. Observations were documented using an observation guide and field notes. Observations were made concerning the home environment, parent-child interaction and medication storage relating to seasonal influenza prevention. The interview and observation guide were developed after a review of relevant literature and discussion with two researchers, colleagues and school teachers experienced in child and family health promotion. A validation process occurred using academic experts, community nurses and school teachers who were knowledgeable about infection control, child and family health and preschool education. This validation process ensured that the instrument was culturally appropriate, explicit, technically correct and able to provide sufficient information to address the proposed research questions (Polit and Beck 2006).

\section{Data Analysis}

A thematic analysis was employed to examine, categorize, tabulate the interview data to address the study objectives (Yin 2014). First, the transcripts from the 20 semi-structured interviews were 
reviewed to mark and link primary codes according to the research propositions. These codes were clustered into broad categories through repetitive reading of the data (Yin 2014). The categories were then grouped into thematic clusters. Analysis continued until no new themes emerged (Yin 2012; Morse and Field 2002; Creswell 2007). Data saturation was observed when similar meanings and categories were noted from the data after the eighteenth interview. Two additional interviews were further finished to confirm that no new data were provided by the parents. A structured approach was taken to establish patterns across these data (Yin 2012). The first author (WL) returned to the data, re-reading numerous times to make comparisons with previously outlined predefined patterns to ensure consistency with the study objectives and identify data that may have been missed. The other authors ( $\mathrm{AD}$ and $\mathrm{CF}$ ) independently assessed the identified themes and data extraction process and consensus was reached on the findings thereby ensuring rigour (Yin 2014).

The demographic data, observations and field notes revealed culturally related health promotion practices used by Hong Kong Chinese parents in seasonal influenza prevention. These data enriched the qualitative analysis of individual cases and provided a family context for the parent responses.

In a last stage, the families' demographic data, home settings, level of parental education, living environment, and culture related health promotion practices were compared comprehensively across cases. This cross-case analysis provided a thorough understanding of the similarities and variances between families regarding health practices for seasonal influenza prevention (Creswell 
2007; Yin 2014). The concordance between the interview and observation data enhanced the rigor of the findings (Yin 2012) .

\section{Ethical Considerations}

Ethical approval was obtained from the Hong Kong Polytechnic University Human Research and Ethics Committee and University of Technology Sydney Human Research Ethics Committee Human Research and Ethics Committees. Participants also consented to the publication and reporting of individual de-identified data. Parents voluntarily joined the study and were informed that they could withdraw from the research at any time. All data were de-identified during the transcription process.

\section{Findings}

Twenty Hong Kong Chinese parents agreed to be interviewed. They were all mothers, aged between 28-and-45 years and the main caregivers of their preschool children. Ten mothers completed secondary school education. Ten mothers had finished tertiary education. The results show that education level was not a factor affecting mothers' choices of health practices in flu prevention. Mothers who had a secondary or tertiary education chose to use different cultural practices to prevent seasonal influenza. Fifteen mothers were housewives. Five were full-time employees and their children were cared for by other family members. Nine families had three generations: grandparent, parent and child living in the same household. Eleven families had two generations: parent and child living in the same household.

The qualitative analysis of the interviews identified five major cultural practices to prevent seasonal influenza. These practices were: sharing beds; boiling white vinegar to kill air-born germs 
in the air; diet therapy to enhance health; self-prescribed Chinese medication to manage child's flu symptoms and the co-use of Western health products; and traditional Chinese medication to prevent seasonal influenza. The data gleaned from the observations enriched the interview findings.

\section{Sharing Beds}

Many children sleep with their parents, domestic helpers or siblings even when they are ill. The following two examples illustrated this theme.

No matter whether my child is sick or not, she still sleeps with me. If I am sick, it's impossible for me to wear a mask to sleep. She likes to stick and sleep with me. (P8-M22)

Elder daughter sleeps with her dad and younger son sleeps with me. I won't specifically sleep separately with them when I get flu. It's the same. (P11-M23)

The findings of the above quotes were consistent with the situations observed during the home interviews. The household surface area of the 20 participating families ranged from 30-to100 meters. The total number of people living in the household was from four-to-eight people.

Three mothers reported that they would allow their children to sleep alone in their bed as usual when their children developed flu symptoms. Seventeen mothers claimed that sharing a bed was their usual practices no matter whether their children were sick or not sick. Mothers reported that bed sharing makes it easier for them to take care of their child, particularly when the child was sick. Among these 17 mothers, 13 mothers claimed that they sleep with their children. One mother 
said her son shares a bed with his elder brother. Three mothers stated their children slept with the domestic helper.

[Table 1 near here]

\section{Boiling White Vinegar to Kill Air-Born Germs}

Parents reported that they boiled white vinegar to ensure the environment was healthy for their children. Parents claimed they followed other people in using this practice even though they stated they did not know whether it worked or not.

I boiled white vinegar for the effect of disinfection. It will kill the bacteria in the air. When it is peak season of avian flu, flu etc., we follow other friends to boil the vinegar. People say that we can put the boiled vinegar near the window and then let it evaporate in the air. Let wind blow it around the flat. It gets no harm and why not to try. I just boil few times in a year. (P6-M81)

Emm...Yes, boiling white vinegar can kill germs. This kind of way is very common back in the days. In the serious time of flu infection, all white vinegars were sold out. Ha...ha... Even no one proves that boiling white vinegar is an effective way in killing germs. I still will do it. It's better than having nothing done. (P15-M213)

\section{Diet Therapy to Enhance Health}


Parents reported that they made different types of Chinese soups to enhance health using boiling products such as: crocodile meat soup and soup comprised of the lungs of a pig, the Glabrous Greenbrier Rhizome and Ching Bo Leung..

I usually boil Chinese soup. I like Glabrous Greenbrier Rhizome most because it clears away the heat and detoxifies the waste products from our bodies. (P1-M59)

I always ask my mum to boil like Ching Bo Leung to remove heat from our bodies... Those soups help you expel the dampness out from our body. Before, children got a cough for a certain period; grandmother then boiled crocodile meat soup for them since this soup helps to relieve cough. (P3-M355)

We usually boil soup with dried vegetables and pig's lung for them to drink. Pig's lung is used to clear the heat from our bodies as well as moisturize our lungs. (P11-M192)

The above quotes demonstrate that mothers integrated the principle of Traditional Chinese Medicine in their daily diet regime to maintain health. Parents also reported that different Chinese soups have functions such as to clear heat and dampness from the body these were purported as maintaining a balance of heat and cold inside the body.

\section{Self-Prescribed Chinese Medication to Managing a Child's Flu Symptoms}

The most common health practices parents described to manage their children's flu symptoms were the use of self-prescribed Chinese medication such as Monkey Bezoar Powder and Bo Yi Dan. 
This is believed to also prevent seasonal influenza. Monkey Bezoar Powder, also known by its Latin name Calculus Macacae Mulattae powder, is used to clear away excessive phlegm, to relieves coughing and improve breathing.

I will let my younger son take Monkey Bezoar Powder which is kidney stone of monkey mixing it with some Chinese herbs. After taking one time of Rhesus Macaque Bezoar, his cough diminished a lot. He becomes energetic. Sometimes, after taking some western medications, he feels very sleepy and may sleep for a whole day. (P18-M173)

I will give them Bo Ying Dan to eat when their colds symptoms are minor. I will let them take for consecutive two days if they sneeze and have runny nose. They seldom need to consult the doctor after eating Bo Ying Dan. (P20-M116)

\section{Co-Use of Western Health Products and Traditional Chinese Medication to Prevent Seasonal Influenza}

Parents reported that they applied different methods from different perspectives to prevent seasonal influenza. The most often cited approach was the use of both Western health products such as vitamin $\mathrm{C}$ and cod liver oil and Chinese medications such as herbal tea and Bo Ying Dan (a pill to treat fever, sneezing, cough, restlessness and crying at night). These Chinese medicines are thought to dispel wind, clear away heat and eliminate phlegm.

I usually give them vitamins $\mathrm{C}$, cod liver oil etc. to eat to increase their body resistant. I buy and try various healthy products. (P1-M45) 
Children take vitamin C, fish oil and blueberry [for better eye sight] as supplements every day. All are for better body resistance. (P3-M249)

Parents also claimed that they drank herbal tea to promote health and prevent influenza.

Not only for treating flu, you can drink herbal tea in normal days for flu prevention. When children have flu, they will usually drink herbal tea. It is already enough to help them recover from flu. (P13-M239)

Parents gave their children Bo Ying Dan to enhance their body's resistance and prevent seasonal influenza.

After he starts going to school, I let him eat Bo Ying Dan even he's not sick. The purpose of taking it is to increase his body resistance. (P18-M188)

During the home interviews, Western health products such as vitamin C and bilberry capsules and Chinese medications such as Bo Ying Compound and Pure Pearl Powder were observed in three households. Table 2 outlines the medications that were observed in participants' homes. [Table 2 near here]

\section{Discussion}

The findings from this study demonstrates that traditional cultural health practices and beliefs continue to have a strong influence on Chinese Hong Kong parents' practices in managing flu 
symptoms and prevention of seasonal influenza. Parents draw from both traditions in their prevention and health promotion practices in their attempt to maintain the health of their families. The results provide insights about family use of Western and Chinese medicine and can assist community nurses in planning and developing community disease prevention and health promotion programmes with Chinese communities.

\section{Pluralistic Health Promotion approach to Better Prevent Seasonal Influenza}

This study adds to the body of knowledge concerning medical pluralism within Hong Kong society. The findings support that the concurrent use of both Western and Chinese medicines remains the preferred practice of Hong Kong families as found in other research studies (for example see Chung et al. 2007). Two studies (Jamieson, Parker, and Richards 2008; Huber and Shapiro 2012) provide additional support to the findings of this research showing that the health promotion strategies designed to improve health outcomes among Hong Kong Chinese families may be more effective if they are culturally appropriate and family focused . There are limited studies that consider the use of alternative medicine and how it may influence decision-making about seasonal influenza prevention practices. These studies on vaccination uptake include one by Bleser et. al. (2016) who reported that the influenza vaccination uptake rate is lower among children whose families practiced alternative medicine, hence health promotion targeting these families may be useful. Using culturally appropriate seasonal influenza prevention practices and family focused approaches has the potential to encourage family acceptance of and compliance with evidence based health practices that aim to improve influenza management and reduce transmission. 
A review of preparedness plans for seasonal influenza pandemics in Hong Kong found that culturally based problem-solving perspectives and values of the local public are not routinely incorporated into these health strategy plans to prevent seasonal influenza (Centre of Health Protection of Hong Kong 2014b). The Hong Kong Department of Health takes a strategic approach similar to that of many European countries, that focuses on health surveillance and harnessing and deploying available resources such as building up an adequate stock of antiviral agents and vaccines for influenza preparedness and risk reduction (Coker and Mounier-Jack 2006; Department of Health of Hong Kong 2015). Failing to act and incorporate family practices may result in culturally incongruent seasonal influenza preventive measures (Paton et al. 2008; Leininger 2002). Health departments must acknowledge socio-cultural practices and include family members in the development and management of seasonal influenza health promotion initiatives in order to make them appropriate and responsive (Gray et al. 2012).

Our study raises questions concerning what professionals are the most appropriate to advise families as part of the public health response to influenza epidemics in Hong Kong. Mothers in our study reported using Traditional Chinese medicine or seeking the advice of Traditional Chinese Medicine practitioners or herbalist as their first point of contact. Seasonal influenza could be better prevented and treated with improved planning, training and coordination between Western medicine, nursing and Traditional Chinese Medicine practitioners. This will require additional training for Traditional Chinese Medicine practitioners with an emphasis on referral and reporting cases of influenza to enable government surveillance systems to detect the cases at the on-set of an epidemic. This acknowledgement of medical pluralism may help to improve health promotion initiatives to prevent seasonal influenza. 


\section{Nurse-Parent Partnerships to Enable Cultural Congruent Health Promotion}

As with many cultural practices, regardless of the country of origin, they are often neglected by the research community and lack evidence of efficacy. Importantly, until this research is conducted a respectful approach is necessary to avoid the alienation of parents and enable them to speak freely about the interventions they are using with their children and other family members. Involving the health sector and families in health is a fundamental goal of family health promotion (Cyril, Smith, and Renzaho 2015). Yet, an expert medical model remains the preferred way of working for many nurses (and other health professionals) (Fowler, Lee, et al. 2012). Continuing to use this model frequently results in feelings of frustration when the advice provided is not acted upon by the parent.

It is well accepted that people are more likely to take appropriate action and accept suggested actions if they have been engaged in the decision-making process (Gray et al. 2012) and their values have been respected during the programme development (Allmark and Tod 2006). This approach enables the nurses and community health professionals to draw on the parent's intimate knowledge of their family's needs and behaviours, and their cultural preferences (Fowler, Lee, et al. 2012).

Partnerships between community health nurses and families provide opportunities to strengthen the ability of parents to create healthy households through the development of advice and strategies that are contextual and meet the cultural needs of the parent and their family (Gray et al. 2012; Hopwood et al. 2013). Nurses, if provided with sufficient organisational support and professional development, can work collaboratively with families and provide health advice in ways that are 
meaningful and culturally appropriate. A critical skill is the ability to develop a conversation with the parent and respectfully challenge parents' cultural beliefs when needed (Fowler, Rossiter, et al. 2012). This conversation could include explanations of the efficacy or lack of efficacy of specific cultural health practices; to assist parents to set achievable goals and provide individualized health information to address each family's concerns. It is vital for health professionals to foster relationships with parents by engaging parents in conversations about health promotion (Riesch, Anderson, and Krueger 2006). Health professionals must be proactively involved discussing culturally specific health practices such as the use of traditional Chinese medication and bed sharing practices. Such discussions provide opportunities for prompt and appropriate health recommendations that could ensure effective prevention, management and treatment of influenza. For example, if the bedroom space is adequate, separate sleeping practices must be encouraged to minimize prolonged and close exposure of the parent to an infected child (Cowling et al. 2009). These approaches support the most favourable environment for health promotion and illness/disease prevention at both community and household levels.

In our findings, several families had recruited domestic helpers to take care of their child; this is a common practice. In such cases, health professionals should involve these workers and consider using non-written materials or language appropriate teaching materials during outreach health consultation visits.

\section{Conclusion}

This study provides valuable insight into the cultural practices of Chinese Hong Kong mothers in the prevention of seasonal influenza and its treatment. The results of this study demonstrate that 
the traditional cultural health practices and beliefs strongly influence Hong Kong Chinese parents' practices in managing symptoms associated with the common cold and the prevention of seasonal influenza. Parents draw from both Western and Chinese traditions in their prevention and health promotion practices in their attempt to maintain the health of their families. The findings recommended that a pluralistic health promotion approach should be carefully considered in local seasonal influenza prevention programme to ensure a family's acceptance and compliance to the health advice from the health authority. Developing a nurse-parent partnership is an important consideration when planning and implementing culturally appropriate health promotion services in a local context.

\section{Limitations}

In this study, the gender of the participants was homogeneous as all were female. This is an important factor to be considered in future research regarding gender differences in father's health promotion practice and experience. Extending the current research to include diverse samples in ethnicity and/or socio-economic class would be a valuable next step.

Acknowledgements: We would like to thank all participants for their participations to the study.

Conflict of Interest: The authors declare that they have no conflict of interests.

\section{Funding: None}

\section{References}


Adair, P. M., C. M. Pine, G. Burnside, A. D. Nicoll, A. Gillett, S. Anwar, Z. Broukal, et al. 2004. "Familial and cultural perceptions and beliefs of oral hygiene and dietary practices among ethnically and socioeconomical diverse groups." Community Dent Health 21 (1 Suppl):102-11.

Allmark, Peter, and Angela Tod. 2006. "How should public health professionals engage with lay epidemiology?" Journal of Medical Ethics 32 (8):460-3.

Bodrovea, E. , and D.J. Leong. 2007. Tools of the mind: the Vygotskian approach to early childhood education 2nd ed. New Jersey: Pearson.

Centre for Health Promotion of Hong Kong. 2010. "Strategic plan for 2010-2014." In. Hong Kong: Hospital Authority.

Centre of Health Protection of Hong Kong. 2016. "Hand hygiene an easy and effective way to prevent infection.", Accessed 17 Jan http://www.chp.gov.hk/en/exhibition details/2587/0.html

- - . 2014b. "Preparedness Plan for Influenza Pandemic The Government of the Hong Kong Special Administrative Region."

- 2016 . "Seasonal Inflenza ", Accessed 26 January http://www.chp.gov.hk/en/view content/14843.html.

Chan, Siu Mui, J Bowes, and S Wyver. 2009. "Chinese parenting in Hong Kong: Links among goals, beliefs and styles." Early Child Development and Care 179 (7):849-62.

Chen, Y. C. 2001. "Chinese values, health and nursing." Journal of Advanced Nursing 36 (2):270-3.

Christensen, Pia. 2004. "The health-promoting family: a conceptual framework for future research." Social Science \& Medicine 59 (2):377-87. doi: http://dx.doi.org/10.1016/j.socscimed.2003.10.021.

Coker, Richard. , and Sandra Mounier-Jack. 2006. "Pandemic influenza preparedness in the Asia-Pacific region." The Lancet 368 (9538):886-9. doi: http://dx.doi.org/10.1016/S0140-6736(06)69209-X.

Cowling, BJ, KH Chan, VJ Fang, CK Cheng, RO Fung, W Wai, J Sin, WH Seto, R Yung, and DW Chu. 2009. "Facemasks and hand hygiene to prevent influenza transmission in households: a cluster randomized trial." Ann Intern Med 151 (7):437-46.

Cresswell, J.W. 2009. Qualitative, quantitative and mixed methods approaches 2 nd ed. Thousand Oaks: Sage Publications.

Creswell, J.W. 2007. Qualitative inquiry \& research design. 2nd ed. Thousand Oaks Sage Publications

Cyril, Sheila, Ben J. Smith, and Andre M. N. Renzaho. 2015. "Systematic review of empowerment measures in health $\quad$ promotion." $\quad$ Accessed $10 \quad$ Nov. http://heapro.oxfordjournals.org/content/early/2015/07/01/heapro.dav059.abstractN2

Department of Health of Hong Kong. 2015. "Protect yourself from seasonal influenza " In, edited by Hong Kong Department of Health. Hong Kong Hong Kong Department of Health.

Fowler, Cathrine, Alison Lee, Roger Dunston, M Chiarella, and C Rossiter. 2012. "Co-producing parenting practice: learning how to do child and family health nursing differently." Australian Journal of Child and Family Health Nursing 9 (1):7.

Fowler, Cathrine, Chris Rossiter, Margaret Bigsby, Nick Hopwood, Alison Lee, and Roger Dunston. 2012. "Working in partnership with parents: the experience and challenge of practice innovation in child and family health nursing." Journal of Clinical Nursing 21 (21-22):3306-14.

Gray, Lesley, Carol MacDonald, Brenda Mackie, Douglas Paton, David Johnston, and Michael G Baker. 2012. "Community responses to communication campaigns for influenza A (H1N1): a focus group study." BMC Public Health 12 (1):205.

Hofstetter, AnnikaM, Angela Barrett, and MelissaS Stockwell. 2015. "Factors Impacting Influenza Vaccination of Urban Low-Income Latino Children Under Nine Years Requiring Two Doses in the 2010-2011 Season." Journal of Community Health 40 (2):227-34. doi: 10.1007/s10900-014-9921z.

Hong Kong Census and Statistics Department. 2015. "Annual Report." Accessed 28 Oct. http://www.censtatd.gov.hk/hkstat/sub/so20.jsp. 
Hopwood, N., C. Fowler, A. Lee, C. Rossiter, and M. Bigsby. 2013. "Understanding partnership practice in child and family nursing through the concept of practice architectures." Nurs Inq 20 (3):199-210. doi: 10.1111/nin.12019.

Huber, J.T., and R.O. \& Gillaspy Shapiro, M.L. . 2012. "Top down versus bottom up: the social construction of the health literacy movement " Library Quarterly 82 (4):429-51.

Jamieson, L. M., E. J. Parker, and L. Richards. 2008. "Using qualitative methodology to inform an Indigenous-owned oral health promotion initiative in Australia." Health Promotion International 23 (1):52-9.

Jennings, Lance C., Arnold S. Monto, Paul K. S. Chan, Thomas D. Szucs, and Karl G. Nicholson. 2008. "Stockpiling prepandemic influenza vaccines: a new cornerstone of pandemic preparedness plans." The Lancet Infectious Diseases 8 (10):650-8. doi: http://dx.doi.org/10.1016/S14733099(08)70232-9.

Joo, J. Y. 2014. "Effectiveness of culturally tailored diabetes interventions for Asian immigrants to the United States: a systematic review." Diabetes Educ 40 (5):605-15. doi: 10.1177/0145721714534994.

Kwong, Enid Wai-yung, Ivy Oi-yi Lam, and Tony Moon-Fai Chan. 2009. "What factors affect influenza vaccine uptake among community-dwelling older Chinese people in Hong Kong general outpatient clinics?" Journal of Clinical Nursing 18 (7):960-71. doi: 10.1111/j.13652702.2008.02548.x.

Lam, T.P. . 2001. "Strengths and weaknesses of traditional Chinese medicine and western medicine in the eyes of some Hong Kong Chinese " Journal of Epidemiology and Community Health 55:762-5.

Lau, J.T.F., Doreen W. H. Au, H. Y. Tsui, and K. C. Choi. 2012. "Prevalence and determinants of influenza vaccination in the Hong Kong Chinese adult population." American Journal of Infection Control 40 (7):e225-e7. doi: http://dx.doi.org/10.1016/j.ajic.2012.01.036.

Lau, T.F., P.C. Leung, E.L.Y. Wong, C. Fong, K.F. Cheng, S.C. Zhang, C.W.K. Lam, V. Wong, K.M. Choy, and W.M. Ko. 2005. "Using herbal midicine as a means of prevention experience during the SARS crisis " The American Journal of Chinese Medicine 33 (3):345-56.

Leininger, Madeleine. 2002. "Culture Care Theory: A Major Contribution to Advance Transcultural Nursing Knowledge and Practices." Journal of Transcultural Nursing 13 (3):189-92. doi: 10.1177/10459602013003005.

Liao, Q., B. J. Cowling, W. W. Lam, and R. Fielding. 2011. "Factors affecting intention to receive and selfreported receipt of 2009 pandemic (H1N1) vaccine in Hong Kong: a longitudinal study." PLoS One 6 (3):e17713. doi: 10.1371/journal.pone.0017713.

Liu, Xianchen, Lianqi Liu, and Ruzhan Wang. 2003. "Bed sharing, sleep habits, and sleep problems among Chinese school-aged children." Sleep 26 (7):839-44.

Lohiniva, Anna-Leena, Amal Barakat, Erica Dueger, Suzanne Restrepo, and Rajae El Aouad. 2014. "A Qualitative Study of Vaccine Acceptability and Decision Making among Pregnant Women in Morocco during the A (H1N1) pdm09 Pandemic." PLoS One 9 (10):e96244. doi: 10.1371/journal.pone.0096244.

Ludman, Elaine Kris, and Jacqueline M Newman. 1984. "Yin and yang in the health-related food practices of three Chinese groups." Journal of Nutrition Education 16 (1):3-5.

Morse, J. M, and P. A Field. 2002. Qualitative research methods for health professionals 3rd ed. Thousand Oaks: Sage Publications.

Paton, Douglas, Bruce Parkes, Michele Daly, and Leigh Smith. 2008. "Fighting the flu: Developing sustained community resilience and preparedness." Health Promotion Practice 9 (4 suppl):45S-53S.

Paul, I. M., J. Beiler, A. McMonagle, M. L. Shaffer, L. Duda, and C. M. Berlin, Jr. 2007. "Effect of honey, dextromethorphan, and no treatment on nocturnal cough and sleep quality for coughing children 
and their parents." Arch Pediatr Adolesc Med 161 (12):1140-6. doi: 10.1001/archpedi.161.12.1140.

Polit, Denise F., and Cheryl Tatano Beck. 2006. "The content validity index: Are you sure you know what's being reported? critique and recommendations." Research in Nursing \& Health 29 (5):489-97. doi: 10.1002/nur.20147.

Riesch, S. K., L. S. Anderson, and H. A. Krueger. 2006. "Parent-child communication processes: preventing children's health-risk behavior." J Spec Pediatr Nurs 11 (1):41-56. doi: 10.1111/j.17446155.2006.00042.x.

Stake, R.E. 1995. The art of case study research. London: SAGE.

Stockwell, Melissa S, Annika M Hofstetter, Nathalie DuRivage, Angela Barrett, Nadira Fernandez, Celibell Y Vargas, and Stewin Camargo. 2015. "Text message reminders for second dose of influenza vaccine: a randomized controlled trial." Pediatrics 135 (1):e83-e91.

World Health Organization. 2009. Infection-control measures for health care of patients with acute respiratory diseases in community settings: trainer's guide Geneva: WHO.

Yamamoto, Yoko, and Jin Li. 2012. "What makes a high-quality preschool? Similarities and differences between Chinese immigrant and European American parents' views." Early Childhood Research Quarterly 27 (2):306-15. doi: http://dx.doi.org/10.1016/j.ecresq.2011.09.005.

Yang, Chang-Kook, and Hong-Moo Hahn. 2002. "Cosleeping in young Korean children." Journal of Developmental \& Behavioral Pediatrics 23 (3):151-7.

Yin, R. 2012. Application of case study research 3rd ed. Thousand Oaks: SAGE.

-- - 2014. Case study research: design and methods 5th ed. Los Angeles: SAGE.

Zijtregtop, E. A., J. Wilschut, N. Koelma, J. J. Van Delden, R. P. Stolk, J. Van Steenbergen, J. Broer, B. Wolters, M. J. Postma, and E. Hak. 2009. "Which factors are important in adults' uptake of a (pre)pandemic influenza vaccine?" Vaccine 28 (1):207-27. doi: 10.1016/j.vaccine.2009.09.099. 